\title{
A cautionary tale: the non-causal association between type 2 diabetes risk SNP, rs7756992, and levels of non-coding RNA, CDKAL1-v1
}

\author{
Jonathan M. Locke • Fan-Yan Wei • Kazuhito Tomizawa • \\ Michael N. Weedon • Lorna W. Harries
}

Received: 16 October 2014 / Accepted: 9 January 2015 / Published online: 30 January 2015

(C) The Author(s) 2015. This article is published with open access at Springerlink.com

\begin{abstract}
Aims/hypothesis Intronic single nucleotide polymorphisms (SNPs) in the CDKAL1 gene are associated with risk of developing type 2 diabetes. A strong correlation between risk alleles and lower levels of the non-coding RNA, CDKAL1-v1, has recently been reported in whole blood extracted from Japanese individuals. We sought to replicate this association in two independent cohorts: one using whole blood from white UK-resident individuals, and one using a collection of human pancreatic islets, a more relevant tissue type to study with respect to the aetiology of diabetes.

Methods Levels of CDKAL1-v1 were measured by real-time PCR using RNA extracted from human whole blood $(n=70)$ and human pancreatic islets $(n=48)$. Expression with respect to genotype was then determined.

Results In a simple linear regression model, expression of CDKAL1-v1 was associated with the lead type 2 diabetesassociated SNP, rs7756992, in whole blood and islets. However, these associations were abolished or substantially reduced in multiple regression models taking into account rs9366357 genotype: a moderately linked SNP explaining a much larger amount of the variation in CDKAL1-v1 levels, but not strongly associated with risk of type 2 diabetes.
\end{abstract}

Electronic supplementary material The online version of this article (doi:10.1007/s00125-015-3508-9) contains peer-reviewed but unedited supplementary material, which is available to authorised users.

J. M. Locke • M. N. Weedon • L. W. Harries $(\bowtie)$

Institute of Biomedical and Clinical Science, University of Exeter

Medical School, RILD Building, Barrack Road,

Exeter EX2 5DW, UK

e-mail: 1.w.harries@exeter.ac.uk

F.-Y. Wei $\cdot$ K. Tomizawa

Department of Molecular Physiology, Faculty of Life Sciences, Kumamoto University, Kumamoto, Japan
Conclusions/interpretation Contrary to previous findings, we provide evidence against a role for dysregulated expression of $C D K A L 1-v 1$ in mediating the association between intronic SNPs in $C D K A L 1$ and susceptibility to type 2 diabetes. The results of this study illustrate how caution should be exercised when inferring causality from an association between diseaserisk genotype and non-coding RNA expression.

Keywords $C D K A L 1$ - Expression quantitative trait loci · Genome-wide association studies · Non-coding RNA · Type 2 diabetes
Abbreviations
eQTL Expression quantitative trait loci
GWAS Genome-wide association studies
LD Linkage disequilibrium
$\mathrm{ms}^{2} \quad$ 2-Methylthio
SNP Single nucleotide polymorphism
SR Serine/arginine-rich

\section{Introduction}

One of the most robust associations between common genetic variation and type 2 diabetes risk, reported in European and Asian populations, involves intronic single nucleotide polymorphisms (SNPs) in the CDKAL1 gene, encoding CDK5 regulatory subunit associated protein 1-like 1 [1]. CDKAL1 encodes a methylthiotransferase that catalyses the 2methylthio $\left(\mathrm{ms}^{2}\right)$ modification of various substrates, including the $\mathrm{ms}^{2}$ addition to $N^{6}$-threonyl-carbamoyladenosine at position 37 of tRNA ${ }^{\text {Lys }}$ (UUU) [2]. The $\mathrm{ms}^{2}$ modification of tRNA $^{\text {Lys }}(\mathrm{UUU})$ stabilises the interaction with its cognate codons, allowing for efficient translation [3]. This is of particular relevance to the beta cell, where correct processing of 
proinsulin to insulin depends on a lysine residue located at the A-chain/C-peptide cleavage site [3]. Indeed $C D K A L 1$ risk allele carriers display an insulin secretory defect that is concomitant with higher levels of proinsulin [4], and beta cell-specific deletion of Cdkall in mice results in glucose intolerance due to reduced insulin secretion and impaired proinsulin conversion [3]. These observations suggest that diabetes-associated risk alleles in humans are likely to reduce CDKAL1 activity.

It has been reported that the type 2 diabetes-associated risk alleles at this locus are associated with lower levels of a noncoding $C D K A L 1$ splice variant, $C D K A L 1-v 1$, which regulates CDKAL1 activity [5]. Zhou et al showed CDKAL1-v1 contains binding sites for a microRNA, miR-494, that also targets the full-length $C D K A L 1$ transcript. By competing for miR-494, CDKAL1-v1 regulates CDKAL1 activity such that if levels of CDKAL1-v1 are lower, less miR-494 is sequestered away from CDKAL1 mRNA and levels of CDKAL1 protein are reduced [5]. Whilst offering a plausible mechanism underlying the type 2 diabetes association, we sought to replicate their findings in another population and a more diseaserelevant tissue type.

\section{Methods}

Participants/nucleic acid extraction The study was carried out in accordance with the Declaration of Helsinki as revised in 2008. Clinical and genetic characteristics are presented in Electronic Supplementary Material (ESM) Table 1. RNA was extracted from whole blood of non-diabetic (all donor $\mathrm{HbA}_{1 \mathrm{c}}$ values $<48 \mathrm{mmol} / \mathrm{mol}$ ) white UK-resident donors using PAXgene Blood RNA Tubes (Qiagen, Venlo, the Netherlands) and PAXgene Blood miRNA Kit (Qiagen). DNA was extracted from EDTA tubes using the Wizard Genomic DNA Purification Kit (Promega, Madison, WI, USA). Snap-frozen pancreatic islets were supplied by ProCell Biotech (Newport Beach, CA, USA) and the National Institute of Diabetes and Digestive and Kidney Disease-funded Integrated Islet Distribution Program at City of Hope (Duarte, CA, USA). RNA was extracted using the mirVana miRNA Isolation Kit (Life Technologies, Carlsbad, CA, USA) and the small amounts of co-eluted genomic DNA whole genome amplified using the REPLI-g Mini Kit (Qiagen).

Genotyping SNPs were genotyped using TaqMan SNP Genotyping Assays (C 30175809_10, rs9366357; C_2504058_20, rs7756992)(Life Technologies) and TaqMan Genotyping Master Mix (Life Technologies).

Quantitative RT-PCR Total RNA was reverse transcribed using the SuperScript VILO Kit (Life Technologies). For real-time PCR, TaqMan Gene Expression Assays (ESM Table 2 presents assay IDs/sequences) and TaqMan Fast Advanced Master Mix (Life Technologies) were used. In islets and in whole blood from UK-resident donors, $C D K A L 1-v 1$ expression was normalised using the geometric mean of five ( $A C T B, B 2 M$, GUSB, HMBS, RPL11) and two (18S, B2M) housekeeping genes, respectively. Expression was calculated using the comparative $C_{t}$ method [6] prior to $\log$ transformation to create parametric data suitable for regression analyses. For all regression analyses involving islet and UK blood samples, expression data were generated using the CDKAL1-v1 assay without an oligonucleotide binding to a sequence overlapping rs 9366357 .

Statistical analysis Regression analyses were performed assuming an additive genetic model. In neither UK whole blood nor islet cohorts were age, sex, BMI or RNA integrity number values associated with $C D K A L 1-v 1$ levels.

\section{Results}

The TaqMan assay (Hs01557326) previously used to quantify CDKAL1-v1 [5] includes an oligonucleotide that binds to a sequence containing the common SNP, rs9366357, which is in moderate linkage disequilibrium (LD) with lead type 2 diabetes-associated SNP, rs7756992 (1000 Genomes Pilot 1: $r^{2}=0.3$, JPT population; $r^{2}=0.28$, CEU population). We therefore wanted to determine whether the expression quantitative trait loci (eQTL) finding could be an artefact of allelic dropout and LD with rs7756992. To address this, we designed another assay, not including primers/probe overlapping rs9366357, to measure CDKAL1-v1. In 70 UK blood samples we found that levels of CDKAL1-v1, quantified using the two TaqMan assays, were very highly correlated $\left(r^{2}=0.93\right)$. Hence, we do not believe that the previous results [5] are affected by allelic dropout.

Having eliminated the possibility that the results from the Japanese study were due to a technical artefact we sought to replicate their correlation between rs7756992 genotype and CDKAL1-v1 levels. Given our sample size of 70, and based on the per-allele effect size observed in the Japanese study, we calculated we had $>95 \%$ power to detect this association (with a type I error rate of 5\%). Indeed, under a simple linear regression model we also found an effect for rs7756992 on CDKA $L 1-v 1$ levels $(\beta=-0.75, p=0.005)$ (Fig. 1, Table 1), but no association with levels of CDKAL1 mRNA $(\beta=0.04$, $p=0.61$ ). Furthermore, in contrast to the previous report [5], there was no correlation between levels of CDKAL1 and CDKAL1-v1 $\left(r^{2}=0.00, p=0.85\right)$.

We remained intrigued by the presence of rs $936635714 \mathrm{bp}$ from the unique splice site of CDKAL1-v1. We used ESEfinder [7] to ascertain whether the alternative alleles of rs9366357 might affect the binding of any serine/argininerich (SR) proteins which may be important for regulating CDKAL1-v1 levels. Indeed the $\mathrm{T}$ allele was predicted to 
Fig. 1 CDKAL1-v1 levels stratified by genotype, $(\mathbf{a}, \mathbf{b})$ in whole blood from 70 white UK-resident donors, $(\mathbf{c}, \mathbf{d})$ in pancreatic islets from 48 white donors and (e) in whole blood from 103 Japanese donors. $y$-axis values were calculated using the comparative $\mathrm{C}_{\mathrm{t}}$ method with values relative to the expression of CDKAL1-v1 in one donor sample
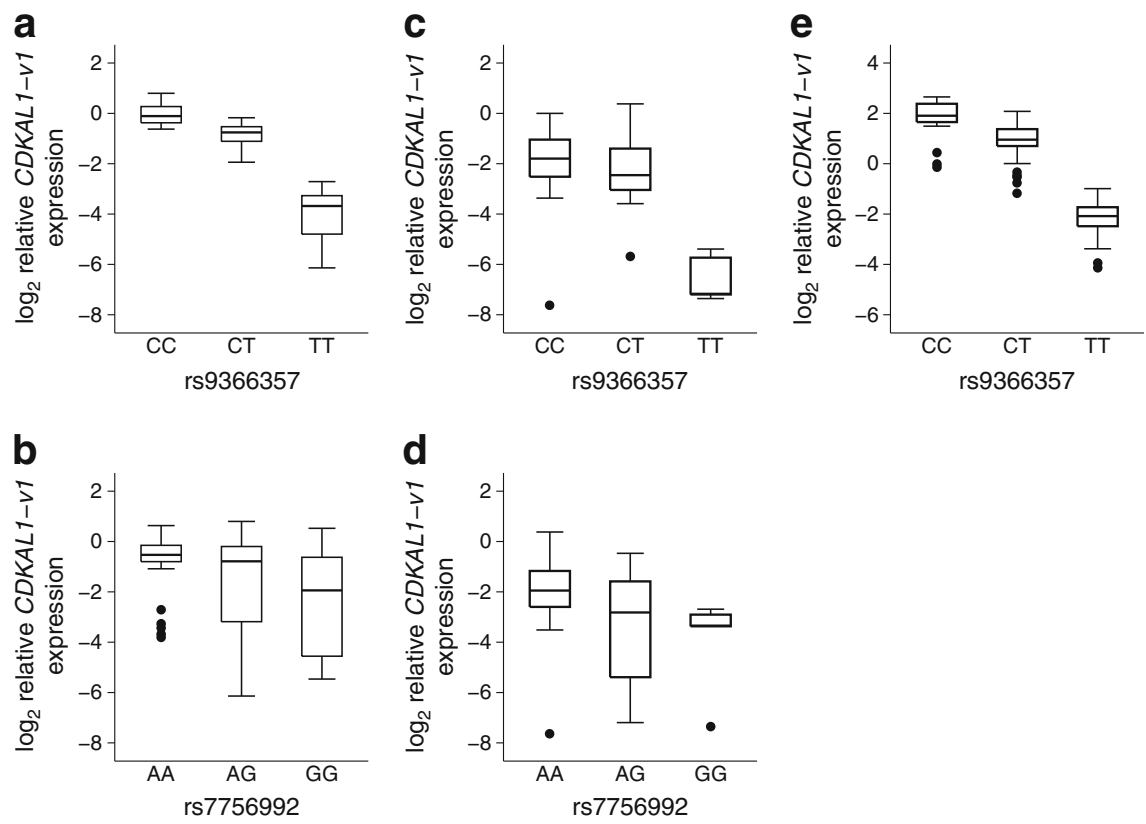

abolish two strong binding sites for the SR protein, SR splicing factor 6 . Given this finding, we determined whether there was any association between rs9366357 and levels of $C D K A$ L1-v1. In the $70 \mathrm{UK}$ blood samples we found an association between rs9366357 and CDKAL1-v1 levels in simple linear regression $\left(\beta=-1.94, p=3.2 \times 10^{-23}\right)$ and in multiple linear regression, taking into account $\operatorname{rs} 7756992(\beta=-1.87$, $\left.p=1.3 \times 10^{-22}\right)$. The lead type 2 diabetes-associated SNP, rs7756992, was still associated with CDKAL1-v1 levels when taking into account rs9366357, although the effect size was reduced and far smaller than the effect of rs9366357 $(\beta=-0.34, p=0.01)$ (Fig. 1, Table 1). Subsequently we genotyped rs9366357 in the 103 Japanese blood samples described in the first report detailing the CDKAL1-v1-rs7756992 association [5]. Again a striking effect of rs9366357 on CDKAL1-v1 levels was seen (multiple linear regression $\beta=-2.12, p=1.9 \times 10^{-25}$ ), but in this data set rs7756992 was no longer associated with
CDKAL1-v1 levels when taking into account rs9366357 $(\beta=-0.07, p=0.60)$ (Fig. 1, Table 1).

We next sought to determine whether CDKAL1-v1 is similarly regulated in human pancreatic islets - the primary tissue of interest with respect to the type 2 diabetes association. In our cohort of 48 islets from white donors we found, in simple linear regression analyses, both $\operatorname{rs} 9366357(\beta=-1.57$, $\left.p=2.4 \times 10^{-4}\right)$ and $\operatorname{rs} 7756992(\beta=-1.07, p=0.009)$ to be associated with $C D K A L 1-v 1$ levels. However, in a multiple regression model only rs9366357 $(\beta=-1.33, p=0.003)$, and not rs7756992 $(\beta=-0.61, p=0.12)$, was associated with $C D K A$ L1-v1 expression (Fig. 1, Table 1).

Lastly, we investigated the association between rs9366357 and type 2 diabetes risk in a large case-control study. In data available from the DIAGRAM consortium, and involving 12,171 cases and 56,862 controls of North European descent, rs9366357 is only very weakly associated with risk of

Table 1 Results of linear regression analyses with CDKAL1-v1 expression as the dependent variable and rs 7756992 and rs9366357 as explanatory variables

\begin{tabular}{|c|c|c|c|c|c|}
\hline \multirow[t]{2}{*}{ Cohort } & \multirow[t]{2}{*}{ SNP } & \multicolumn{2}{|c|}{ Simple linear regression } & \multicolumn{2}{|c|}{ Multiple linear regression } \\
\hline & & $\beta^{\mathrm{a}} \pm \mathrm{SE}$ & $p$ value & $\beta^{\mathrm{a}} \pm \mathrm{SE}$ & $p$ value \\
\hline \multirow[t]{2}{*}{ Whole blood, white UK, $n=70$} & rs7756992 & $-0.75 \pm 0.26$ & 0.005 & $-0.34 \pm 0.13$ & 0.01 \\
\hline & rs9366357 & $-1.94 \pm 0.13$ & $3.2 \times 10^{-23}$ & $-1.87 \pm 0.13$ & $1.3 \times 10^{-22}$ \\
\hline \multirow[t]{2}{*}{ Whole blood, Japan, $n=103$} & rs7756992 & $-1.04 \pm 0.20$ & $1.3 \times 10^{-6}$ & $-0.07 \pm 0.14$ & 0.60 \\
\hline & rs9366357 & $-2.16 \pm 0.13$ & $9.3 \times 10^{-31}$ & $-2.12 \pm 0.15$ & $1.9 \times 10^{-25}$ \\
\hline \multirow[t]{2}{*}{ Whole islets, white, $n=48$} & rs7756992 & $-1.07 \pm 0.39$ & 0.009 & $-0.61 \pm 0.39$ & 0.12 \\
\hline & rs9366357 & $-1.57 \pm 0.39$ & $2.4 \times 10^{-4}$ & $-1.33 \pm 0.42$ & 0.003 \\
\hline
\end{tabular}

${ }^{a}$ Effect size denotes per minor allele change in $\log _{2}$-transformed expression values 
developing type 2 diabetes (OR 1.05, $p=0.003$ ) compared with the lead SNP, rs7756992 (OR 1.20, $p=1.3 \times 10^{-22}$ ). If differential $C D K A L 1-v 1$ expression was heavily involved in mediating the association between variants at the CDKAL1 locus and type 2 diabetes susceptibility, we would expect rs9366357, which explains a much larger amount of the variation in levels of CDKAL1-v1 compared with rs7756992, to be more strongly associated with type 2 diabetes risk. The small effect of rs 9366357 on diabetes risk leads us to conclude that dysregulated expression of $C D K A L 1-v 1$ is unlikely to be the only genotype-dependent defect driving the association between genetic variation at the CDKAL1 locus and type 2 diabetes risk.

\section{Discussion}

Despite simple regression analyses replicating the association between lead genome-wide association study (GWAS) SNP and CDKAL1-v1 levels, our more detailed investigations mean we can provide strong evidence against a causal role for this eQTL. Although eQTL studies provide an important tool for translating GWAS hits, inferring causal mechanisms should be made with caution, particularly with respect to cis-eQTLs involving non-coding RNAs. Probably due to lower functional constraints, cis-eQTL effect sizes are likely to be much larger for non-coding RNAs than for protein-coding transcripts; indeed, this has been reported when comparing cis-eQTLs involving large intergenic RNAs with cis-eQTLs involving protein-coding transcripts [8]. This has the potential, as clearly illustrated by our results, to make non-causal eQTLs appear causal, even if LD between the two SNPs is modest $\left(r^{2}<0.7\right)$.

Given that highly cell type-specific regulation of gene expression is possible, our study may be confounded by the cellular heterogeneity of whole blood and whole islets. Whilst a study of CDKAL1-v1 expression in sorted pancreatic beta cells would be ideal, we consider our analysis unlikely to be strongly confounded: human islets are $\sim 75 \%$ beta cells and $87 \%$ of the variance in beta cell gene expression can be explained by using islet expression as a proxy [9].

Future work should consider the existence of other genes at the $C D K A L 1$ locus when attempting to decipher causal mechanisms. Indeed, whilst physiological characterisation of the biochemical defect strongly implicates CDKAL1 as the causative gene, it has been reported that the associated intronic region contains enhancer elements for the nearby transcription factor, SOX-4, which is important in pancreas development and mature beta cell function [10].

Acknowledgements We would like to thank D. Hogg (University of Exeter Medical School, UK) for his kind technical assistance.
Funding This paper presents independent research funded by the Medical Research Council (grant number MR/J006777/1) and supported by the National Institute for Health Research (NIHR) Exeter Clinical Research Facility. The views expressed are those of the authors and not necessarily those of the Medical Research Council, UK National Health Service, NIHR or the UK Department of Health.

Access to research materials Data is not currently available for public release.

Duality of interest The authors declare that there is no duality of interest associated with this manuscript.

Contribution statement All authors were involved in critical revision of the article, interpretation of the data and approval of the final version for publication. JML, MNW, KT and LWH were involved in the study design. JML, MNW and FYW acquired the data. JML drafted the article. LWH is the guarantor of this work.

Open Access This article is distributed under the terms of the Creative Commons Attribution License which permits any use, distribution, and reproduction in any medium, provided the original author(s) and the source are credited.

\section{References}

1. Mahajan A, Go MJ, Zhang W et al (2014) Genome-wide trans-ancestry meta-analysis provides insight into the genetic architecture of type 2 diabetes susceptibility. Nat Genet 46:234-244

2. Arragain S, Handelman SK, Forouhar F et al (2010) Identification of eukaryotic and prokaryotic methylthiotransferase for biosynthesis of 2-methylthio- $\mathrm{N}^{6}$-threonylcarbamoyladenosine in tRNA. J Biol Chem 285:28425-28433

3. Wei FY, Suzuki T, Watanabe S et al (2011) Deficit of tRNA(Lys) modification by Cdkall causes the development of type 2 diabetes in mice. J Clin Invest 121:3598-3608

4. Dimas AS, Lagou V, Barker A et al (2014) Impact of type 2 diabetes susceptibility variants on quantitative glycemic traits reveals mechanistic heterogeneity. Diabetes 63:2158-2171

5. Zhou B, Wei FY, Kanai N, Fujimura A, Kaitsuka T, Tomizawa K (2014) Identification of a splicing variant that regulates type 2 diabetes risk factor CDKAL1 level by a coding-independent mechanism in human. Hum Mol Genet 23:4639-4650

6. Livak KJ, Schmittgen TD (2001) Analysis of relative gene expression data using real-time quantitative PCR and the 2(-Delta Delta C(T)) Method. Methods 25:402-408

7. Cartegni L, Wang J, Zhu Z, Zhang MQ, Krainer AR (2003) ESEfinder: a web resource to identify exonic splicing enhancers. Nucleic Acids Res 31:3568-3571

8. Popadin K, Gutierrez-Arcelus M, Dermitzakis ET, Antonarakis SE (2013) Genetic and epigenetic regulation of human lincRNA gene expression. Am J Hum Genet 93:1015-1026

9. Nica AC, Ongen H, Irminger JC et al (2013) Cell-type, allelic, and genetic signatures in the human pancreatic beta cell transcriptome. Genome Res 23:1554-1562

10. Ragvin A, Moro E, Fredman D et al (2010) Long-range gene regulation links genomic type 2 diabetes and obesity risk regions to HHEX, SOX4, and IRX3. Proc Natl Acad Sci U S A 107:775-780 